\title{
Aldosterone Rapidly Enhances Levels of the Striatin and Caveolin-1 Proteins in Rat Kidney: The Role of the Mineralocorticoid Receptor
}

\author{
Kevalin Inthachart ${ }^{1}$, Krissanapong Manotham ${ }^{2}$, Somchai Eiam-Ong ${ }^{3}$, Somchit Eiam-Ong ${ }^{4}$ \\ ${ }^{1}$ Interdepartment of Physiology, Chulalongkorn University Graduate School; ${ }^{2}$ Department of Medicine, Lerdsin General \\ Hospital; ${ }^{3}$ Division of Nephrology, Department of Medicine, ${ }^{4}$ Department of Physiology, Chulalongkorn University Faculty of \\ Medicine, Bangkok, Thailand
}

Background: Striatin and caveolin-1 (cav-1) are scaffolding/regulating proteins that are associated with salt-sensitive high blood pressure and promote renal sodium and water reabsorption, respectively. The mineralocorticoid receptor (MR) interacts with striatin and cav-1, while aldosterone increases striatin and cav-1 levels. However, no in vivo data have been reported for the levels of these proteins in the kidney.

Methods: Male Wistar rats were intraperitoneally injected with normal saline solution, aldosterone alone (Aldo: $150 \mu \mathrm{g} / \mathrm{kg}$ body weight), or aldosterone after pretreatment with eplerenone, an MR blocker, 30 minutes before the aldosterone injection (eplerenone [Ep.]+Aldo). Thirty minutes after the aldosterone injection, the amount and localization of striatin and cav-1 were determined by Western blot analysis and immunohistochemistry, respectively.

Results: Aldosterone increased striatin levels by $150 \%(P<0.05)$, and cav-1 levels by $200 \%(P<0.001)$. Eplerenone had no significant effect on striatin levels, but partially blocked the aldosterone-induced increase in cav-1 levels. Aldosterone stimulated striatin and cav-1 immunoreactivity in both the cortex and medulla. Eplerenone reduced cav-1 immunostaining in both areas; however, striatin intensity was reduced in the cortex, but increased in the medulla.

Conclusion: This is the first in vivo study demonstrating that aldosterone rapidly enhances renal levels of striatin and cav-1. Aldosterone increases striatin levels via an MR-independent pathway, whereas cav-1 is partially regulated through MR.

Keywords: Aldosterone; Rapid action; Striatin; Caveolin 1; Protein levels; Rat kidney

\section{INTRODUCTION}

Striatin and caveolin-1 (cav-1), which are scaffolding/regulating proteins in various tissues, play roles in modulating cellular function through multifunctional signals [1,2]. In humans and rodents, single nucleotide polymorphic variants of the gene cod- ing for striatin are associated with salt-sensitive high blood pressure [3]. Therefore, striatin is thought to be present in the kidney. Unfortunately, studies of striatin related to kidney function have not been reported. The cav-1 protein promotes renal water and salt reabsorption via modulation of sodium-chloride cotransporter function and regulation of vascular endothelial ni-
Received: 9 April 2019, Revised: 28 June 2019, Accepted: 1 July 2019

Corresponding author: Somchit Eiam-Ong

Department of Physiology, Chulalongkorn University Faculty of Medicine, 1873 Rama IV, Wangmai, Pathumwan, Bangkok 10330, Thailand

Tel: +66-2-256-4267, Fax: +66-2-256-4267, E-mail: eiamong@yahoo.com
Copyright $(2019$ Korean Endocrine Society

This is an Open Access article distributed under the terms of the Creative Commons Attribution Non-Commercial License (http://creativecommons.org/ licenses/by-nc/4.0/) which permits unrestricted non-commercial use, distribution, and reproduction in any medium, provided the original work is properly cited. 
tric oxide [4].

Aldosterone, a hormone produced by zona glomerulosa cells of the adrenal cortex, regulates sodium, potassium, and acidbase balance, principally in the kidney [5]. In addition to genomic action, which occurs via the aldosterone-mineralocorticoid receptor (MR) complex, aldosterone can also act through rapid ( $\leq 30$ minutes) non-genomic mechanisms that are mediated by MR, or possibly through a membrane- associated pathway [6-10]. This non-genomic process is insensitive to transcription or translation inhibitors [6-10].

MR is localized in epithelial cells of the kidney and gut, and is also found in non-epithelial cells such as neurons and vascular smooth muscle cells [11]. MR has been shown to interact with several proteins, including striatin and cav-1 [2]. A previous in vitro study showed that striatin interacted with MR in mouse aortic endothelial cells, human endothelial cells, and mouse heart tissue [12]. Both in vitro and in vivo investigations have demonstrated that aldosterone increased striatin protein abundance, with a peak at 6 hours and a return to baseline after 12 hours. This effect was found to be inhibited by an MR antagonist [12]. A recent in vitro study reported that striatin was essential in mediating rapid responses to aldosterone [13]. A previous examination of mouse heart tissue found support for the interplay between cav-1 and MR in modulating the mechanisms of aldosterone action [14]. In cultured human umbilical vein endothelial cells, aldosterone incubation for 48 hours induced abundant cav-1 levels [15]. In this regard, aldosterone and MR in the kidney might contribute to the aforementioned effects of both proteins.

Currently, no in vivo data exist on the effects of aldosterone on striatin and cav-1 levels in rat kidney tissue. The present study was conducted to investigate the rapid actions of aldosterone on striatin and cav-1 levels and localization in rat kidney tissue by Western blot analysis and immunohistochemistry, respectively. The role of MR in this relationship was also investigated.

\section{METHODS}

\section{Animals}

Male Wistar rats aged 6 to 8 weeks that weighed 200 to $240 \mathrm{~g}$ and were pathogen-free were obtained from the National Center of Scientific Use of Animals, Mahidol University, Nakornpathom, Thailand. The rats were housed in a room with a controlled temperature $\left(23^{\circ} \mathrm{C} \pm 1{ }^{\circ} \mathrm{C}\right)$ and a 12-hour light/dark cycle. The animals were fed regular rat chow (dry pellets) and tap water ad libitum [16-19]. The rats were acclimatized for 3 days before starting the experiment. All animal protocols were approved by the Ethics Committee of Research, Chulalongkorn University (Permit number IRB 019/2560). The serum creatinine level of each rat was required to be $<1 \mathrm{mg} / \mathrm{dL}$ [16-19].

\section{Experimental design}

The rats were divided into three main groups: sham, aldosterone (Aldo), and eplerenone (Ep.)+Aldo ( $n=6 /$ group). The groups were defined as follows: (1) sham: rats received normal saline solution (NSS) $(0.5 \mathrm{~mL} / \mathrm{kg}$ body weight [BW]) by an intraperitoneal injection; (2) Aldo: rats received an intraperitoneal injection of aldosterone (150 $\mu \mathrm{g} / \mathrm{kg}$ BW; diluted in NSS); (3) Ep.+Aldo: rats received an intraperitoneal injection of eplerenone (an MR blocker) at a dose of $15 \mathrm{mg} / \mathrm{kg}$ BW diluted in dimethyl sulfoxide 30 minutes before the aldosterone injection. This dose of aldosterone was chosen because it was previously used in studies quantifying levels of protein kinase $\mathrm{C}$ alpha $(\mathrm{PKC} \alpha) ; \alpha 1-\mathrm{Na}^{+}$, $\mathrm{K}^{+}$-ATPase; PKC $\beta$ (I and II), and $\mathrm{Na}^{+} / \mathrm{H}^{+}$exchanger proteins (1 and 3) $[18,19]$. Therefore, in the present investigation, we further examined the effect of this dose on striatin and cav-1 levels.

On the day of the experiment, 30 minutes after the NSS or aldosterone injection, rats were anesthetized with an intraperitoneal injection of thiopental (100 mg/kg BW) [17-19]. Plasma samples obtained from the abdominal aorta were stored at $-80^{\circ} \mathrm{C}$ until use for aldosterone level measurements by a radioimmunoassay kit (Aldo-Riact, CIS Bio International, Gif-sur-Yvette, France). Blood and urine chemistry were measured by an indirect method (Model CX3, Beckman, Krefeld, Germany). The kidneys were removed, and half of each kidney was fixed in liquid nitrogen and then stored at $-80^{\circ} \mathrm{C}$ until use for the measurement of striatin and cav-1 levels by Western blot analysis. The other half of the renal tissue was fixed in $10 \%$ paraformaldehyde, subjected to tissue processing by an automated tissue processor (Shandon Citadel 2000, Thermo Scientific, Rockford, IL, USA), and embedded in paraffin wax for localization of these proteins by immunohistochemistry [17-19].

\section{Western blot analysis}

Electrophoresis and immunoblotting were performed as previously described [17-19]. Renal tissue samples were homogenized on ice with a homogenizer (IKA, T25 Basic, Selangor, Malaysia) in homogenizing buffer (20 mM Tris-HCl; pH 7.5, 2 $\mathrm{mM} \mathrm{MgCl} 2,0.2 \mathrm{M}$ sucrose, and $5 \%[\mathrm{v} / \mathrm{v}]$ protease inhibitor cocktail; Sigma-Aldrich, St. Louis, MO, USA). Homogenate samples were subjected to a serial centrifugation to yield plasma membranes [17-19]. Proteins from the plasma membrane (80 to 
$200 \mu \mathrm{g}$ ) were mixed with sample buffer. Proteins (striatin, cav1 , and $\beta$-actin) were then resolved using $8 \%$ to $10 \%$ sodium dodecyl sulfate-polyacrylamide gel electrophoresis and blotted onto nitrocellulose membranes (Bio-Rad, Hercules, CA, USA). The membranes were incubated with a primary monoclonal antibody to striatin (6: sc-136084; 1:500; Santa Cruz Biotechnology, Dallas, CA, USA) [20], cav-1 (7C8: sc-54564; 1:500; Santa Cruz Biotechnology) [21], or $\beta$-actin (Santa Cruz Biotechnology), followed by the respective horseradish peroxidase-linked secondary antibody (Bio-Rad). Immunoreactive proteins were detected by enhanced chemiluminescence (SuperSignal West Pico kit, Thermo Fisher Scientific Inc., Waltham, MA, USA) and documented using a molecular imager ChemiDoc XRS system (Bio-Rad). Each intensity band was quantified using a High Resolution UV and White Light Gel Doc System linked to a computer analysis system (Quantity One version 4.2, Bio-Rad). The intensity ratio of each studied protein to $\beta$-actin was calculated.

\section{Immunohistochemistry}

Detection of protein localization was performed as previously described [17-19]. Paraffin-embedded kidney tissues were cut to $4-\mu \mathrm{m}$-thick sections for examination of striatin and cav-1. Tissue sections were mounted on 3-aminopropyltriethyloxy-saline coated slides (Sigma-Aldrich) and then deparaffinized in xylene and alcohol, with endogenous peroxidase activity quenched in $3 \%$ hydrogen peroxide for 10 minutes. The non-specific binding of antibody was blocked by incubating tissue sections with 5\% normal horse serum (Vector Laboratory, Burlingame, CA, USA) in phosphate buffer solution (PBS-T) (PBS+0.1\% Tween) for 30 minutes at room temperature. The sections were further incubated in primary antibody against striatin $(1: 1,000)$, or cav-1 $(1: 1,000)$ for over 1 hour at room temperature, followed by biotinylated goat anti-mouse-rabbit immunoglobin (Vector Laboratory) for 60 minutes at room temperature. After incubation, tissue sections were rinsed twice for 10 minutes with PBS-T and once for 10 minutes in PBS, and then reacted with ABC-streptavidin horseradish peroxidase complex (Vector Laboratory) for 60 minutes at room temperature. After washing, the slides were reacted for peroxidative activity in 3,3'-diaminobenzidine solution (Sigma-Aldrich) and counterstained with hematoxylin (CV Laboratories, Bangkok, Thailand). As a negative control, the primary antibody was omitted, resulting in negative staining. Three blinded investigators independently examined and scored the intensity of staining on a semi-quantitative five-tiered grading scale from 0 to $4(0=$ negative; $1=$ trace; $2=$ weak; $3=$ mod- erate; $4=$ strong) as previously described [17-19].

\section{Statistical analysis}

The biochemical results and protein levels of striatin and cav-1 were expressed as mean \pm standard deviation. The statistical significance of differences between groups were assessed by analysis of variance with a post hoc comparison by the Tukey test when appropriate. The median staining intensity scores of renal striatin and cav-1 proteins were presented as previously described [17-19]. The Kruskal-Wallis test was used to analyze differences in the median staining intensity scores of the studied proteins among the three groups (sham, Aldo, and Ep.+Aldo). All statistical analyses were performed using SPSS version 22.0 (IBM Corp., Armonk, NY, USA). $P$ values less than 0.05 were considered to indicate statistical significance.

\section{RESULTS}

\section{Blood and urine biochemical data}

As shown in Table 1, plasma aldosterone levels were significantly higher in the Aldo group than in the sham group (sham:

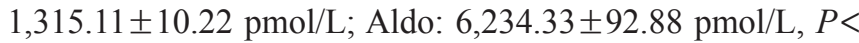
0.001; Ep.+Aldo: 6,288.55 $\pm 87.47, P<0.001)$. There were no significant changes in the levels of any plasma parameters among all studied groups. Neither aldosterone nor eplerenone significantly altered the ratio of plasma sodium to potassium or the ratio of urinary sodium to potassium when compared with the sham group (Table 1).

\section{Aldosterone enhanced renal striatin and cav-1 levels}

By Western blot analysis (Fig. 1), the levels of striatin (110 kDa) and cav-1 (22 kDa) were assessed. Aldosterone significantly elevated the levels of striatin and cav-1 by $149 \% \pm 9 \%(P<0.05)$ and $211 \% \pm 10 \%(P<0.001)$ compared to the sham group, respectively. Eplerenone had no significant effect on striatin levels ( $140 \% \pm 7 \%, P<0.05$ vs. sham, $P=1.00$ vs. Aldo). The increase in cav-1 protein induced by aldosterone was partially blocked by eplerenone, decreasing from $211 \% \pm 10 \%$ to $149 \% \pm 8 \%(P<$ 0.05 vs. sham and vs. Aldo).

\section{Aldosterone activated renal striatin protein localization}

Localization of striatin in the cortex in the sham group is demonstrated in Table 2, Fig. 2B. Immunoreactivity was trace in the glomerulus (GL), proximal convoluted tubule (PCT), distal convoluted tubule (DCT), and peritubular capillary (Pcap). The staining score was weak in the cortical collecting duct (CCD). 
Table 1. Blood and Urine Chemistry in the Experimental Groups

\begin{tabular}{lrrr}
\hline Variable & \multicolumn{1}{c}{ Sham } & Aldo & Ep. + Aldo \\
\hline Plasma aldosterone, $\mathrm{pmol} / \mathrm{L}$ & $1,315.11 \pm 10.22$ & $6,234.33 \pm 92.88^{\mathrm{a}}$ & $6,288.55 \pm 87.47^{\mathrm{a}}$ \\
Plasma sodium, $\mathrm{mmol} / \mathrm{L}$ & $141.32 \pm 1.48$ & $142.17 \pm 6.32$ & $140.22 \pm 4.82$ \\
Plasma potassium, $\mathrm{mmol} / \mathrm{L}$ & $3.53 \pm 0.22$ & $3.58 \pm 0.26$ & $3.49 \pm 0.28$ \\
Plasma chloride, $\mathrm{mmol} / \mathrm{L}$ & $102.64 \pm 1.34$ & $102.31 \pm 2.91$ & $102.42 \pm 2.62$ \\
Plasma bicarbonate, $\mathrm{mmol} / \mathrm{L}$ & $24.71 \pm 1.14$ & $24.29 \pm 1.58$ & $24.48 \pm 1.64$ \\
Plasma creatinine, $\mathrm{mg} / \mathrm{dL}$ & $0.24 \pm 0.02$ & $0.24 \pm 0.02$ & $0.24 \pm 0.03$ \\
Blood urea nitrogen, $\mathrm{mg} / \mathrm{dL}$ & $19.22 \pm 1.64$ & $20.48 \pm 3.28$ & $20.25 \pm 2.68$ \\
Ratio of plasma sodium to potassium & $41.27 \pm 3.64$ & $39.87 \pm 2.94$ & $40.38 \pm 2.87$ \\
Ratio of urinary sodium to potassium & $0.32 \pm 0.02$ & $0.32 \pm 0.02$ & $0.32 \pm 0.03$ \\
\hline
\end{tabular}

Values are expressed as mean \pm standard deviation.

Aldo, aldosterone; Ep., eplerenone ( $n=6 /$ group).

${ }^{\text {a }} P<0.001$ compared with the sham group.

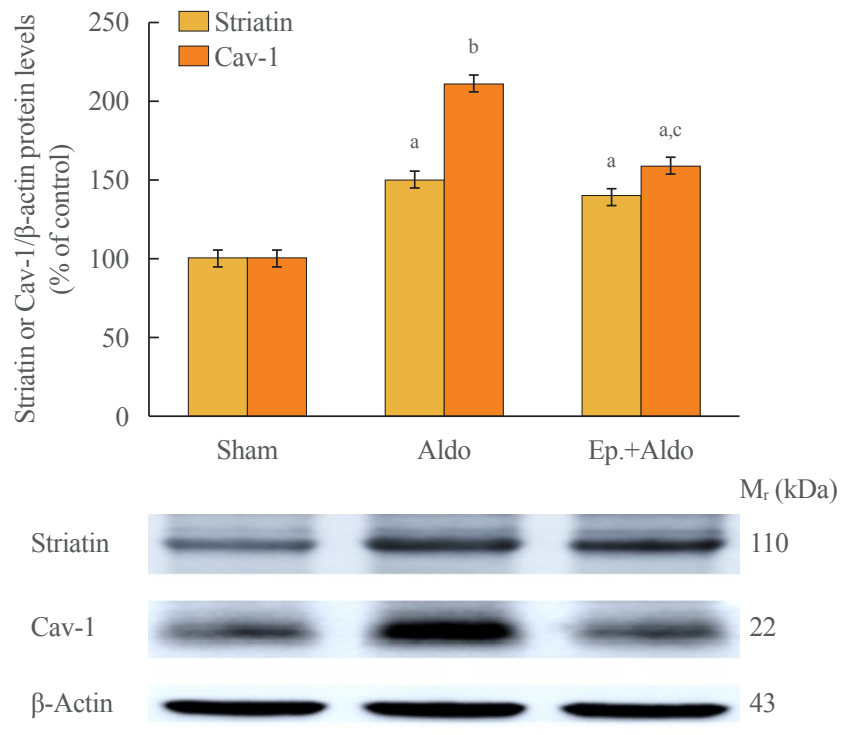

Fig. 1. Effects of aldosterone on renal striatin and caveolin-1 (cav1) protein levels. Representative Western blot analysis of renal striatin and cav-1 protein levels in the sham, aldosterone (Aldo), and eplerenone and aldosterone (Ep.+Aldo) groups ( $n=6 /$ group). Histogram bars show the densitometric ratios of striatin or cav-1 to $\beta$-actin intensity. Data are expressed as mean \pm standard deviation of six independent experiments. ${ }^{\mathrm{a}} P<0.05$; ${ }^{\mathrm{b}} P<0.001$ vs. sham; ${ }^{\mathrm{c}} P<0.05$ vs. Aldo.

Aldosterone increased the intensity score in the GL, CCD, and Pcap to moderate levels (Fig. 2C), whereas immunoreactivity in the PCT and DCT remained weak. Eplerenone reduced the aldosterone-induced increases in the intensity score only in the GL and CCD (Fig. 2D).

In the outer medulla (OM), aldosterone elevated the intensity score from 3 to 4 in the vasa recta (VR) (Table 2, Fig. 2G). Staining in the thick ascending limb of the loop of Henle (TALH), medullary collecting duct (MCD), and thin limb of the loop of Henle (tLH) did not change. Surprisingly, staining intensity scores in all studied areas increased even when pretreatment with eplerenone was applied (Fig. 2H).

In the inner medulla (IM), immunoreactivity was enhanced by aldosterone to strong levels in the MCD and VR, whereas the intensity score in the tLH increased to moderate (Fig. 2K). Eplerenone had no inhibitory effect on the immunoreactivity induced by aldosterone in all studied areas (Fig. 2L).

Table 3 shows the $P$ values obtained by the Kruskal-Wallis test when comparing the median intensity scores of striatin levels among the three treatment groups (sham, Aldo, and Ep.+Aldo) in rat kidney tissue.

\section{Aldosterone altered renal cav-1 localization}

Localization of cav-1 in the cortex in the sham group is shown in Table 2, Fig. 3B. Immunoreactivity was trace in the GL and moderate in the Pcap, and no staining was noted in the PCT, DCT, and CCD. Aldosterone increased immunoreactivity in the GL and Pcap to strong, whereas immunostaining in the PCT, DCT, and $\mathrm{CCD}$ remained at trace levels (Fig. 3C). Eplerenone only reduced the staining score in the GL to moderate (Fig. 3D).

In the OM, aldosterone elevated the intensity score to weak in the TALH, MCD, and tLH (Fig. 3G). These changes were inhibited by pretreatment with eplerenone (Fig. 3H). The intensity score in the VR remained strong in all groups (Fig. 3G, H). In the IM, aldosterone increased the immunoreactivity to a strong level in the VR, but it decreased to a trace level in the tLH (Fig. 3K). 


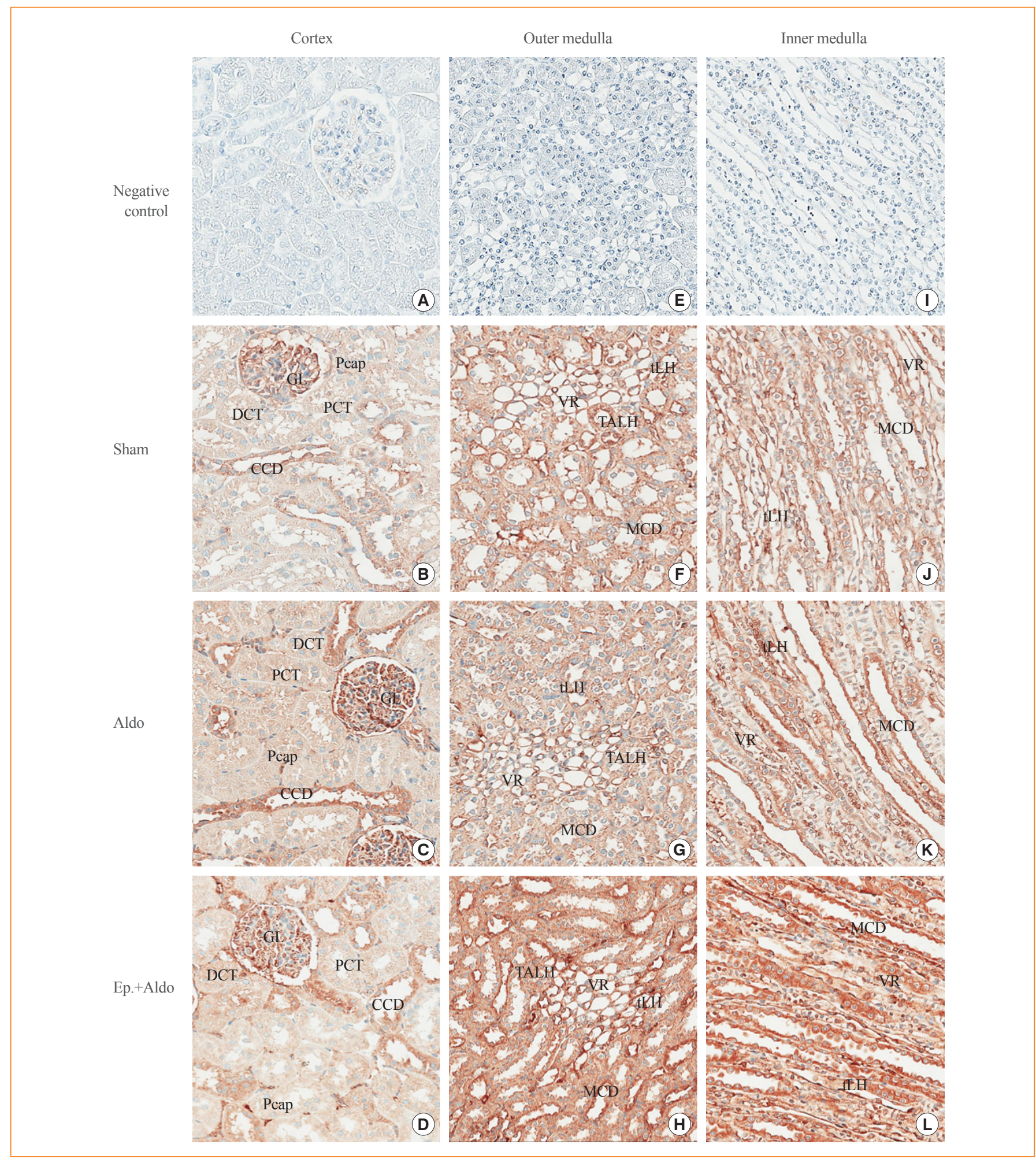

Fig. 2. Effects of aldosterone on renal striatin localization. Representative immunohistochemical staining micrographs of renal striatin in the cortex (A-D), the outer medulla (E-H), and the inner medulla (I-L) from the sham (B, F, J), aldosterone (Aldo) (C, G, K), and eplerenone and aldosterone (Ep.+Aldo) (D, H, L) groups ( $n=6$ /group). Negative controls: A, E, I (A-D, $\times 400$; E-L, $\times 200)$. GL, glomerulus; Pcap, peritubular capillary; DCT, distal convoluted tubule; PCT, proximal convoluted tubule; CCD, cortical collecting duct; tLH, thin limb of the loop of Henle; VR, vasa recta; TALH, thick ascending limb of the loop of Henle; MCD, medullary collecting duct. 


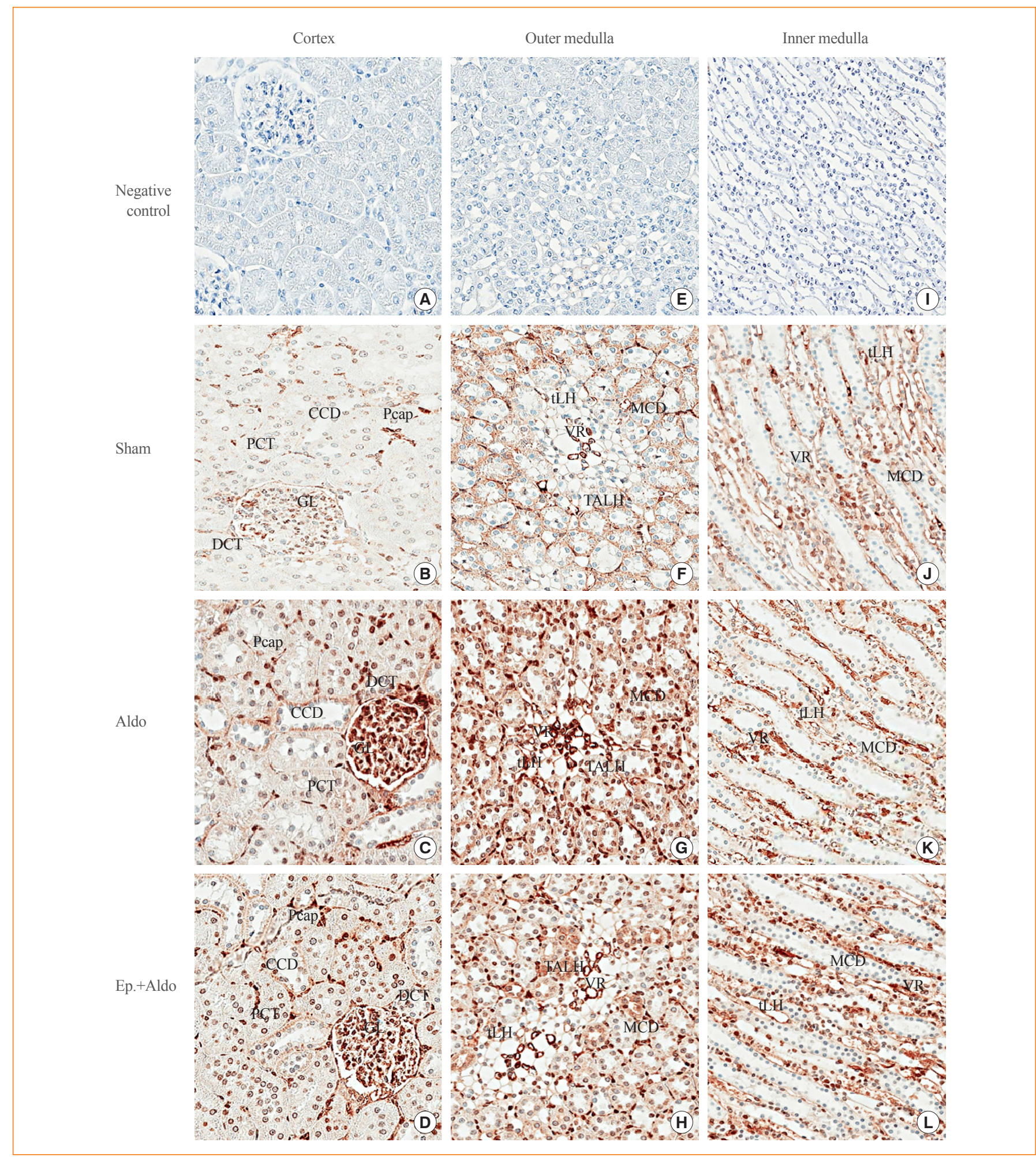

Fig. 3. Effects of aldosterone on renal cav-1 localization. Representative immunohistochemical staining micrographs of renal cav-1 in the cortex (A-D), the outer medulla (E-H), and the inner medulla (I-L) from the sham (B, F, J), aldosterone (Aldo) (C, G, K), and eplerenone and aldosterone (Ep.+Aldo) (D, H, L) groups ( $n=6 /$ group). Negative controls: A, E, I (A-D, $\times 400$; E-L, $\times 200)$. CCD, cortical collecting duct; Pcap, peritubular capillary; PCT, proximal convoluted tubule; GL, glomerulus; DCT, distal convoluted tubule; tLH, thin limb of the loop of Henle; MCD, medullary collecting duct; VR, vasa recta; TALH, thick ascending limb of the loop of Henle. 
Table 2. Median Staining Intensity Scores of Renal Striatin and Caveolin-1 Localization

\begin{tabular}{|c|c|c|c|c|c|c|}
\hline & \multicolumn{3}{|c|}{ Striatin } & \multicolumn{3}{|c|}{ Caveolin-1 } \\
\hline & Sham & Aldo & Ep.+Aldo & Sham & Aldo & Ep.+Aldo \\
\hline \multicolumn{7}{|l|}{ Cortex } \\
\hline GL & 1 & 3 & 2 & 1 & 4 & 3 \\
\hline PCT & 1 & 2 & 2 & 0 & 1 & 1 \\
\hline DCT & 1 & 2 & 2 & 0 & 1 & 1 \\
\hline CCD & 2 & 3 & 2 & 0 & 1 & 1 \\
\hline Pcap & 1 & 3 & 3 & 3 & 4 & 4 \\
\hline \multicolumn{7}{|c|}{ Outer medulla } \\
\hline TALH & 2 & 2 & 3 & 1 & 2 & 1 \\
\hline $\mathrm{MCD}$ & 2 & 2 & 4 & 1 & 2 & 1 \\
\hline VR & 3 & 4 & 4 & 4 & 4 & 4 \\
\hline $\mathrm{tLH}$ & 3 & 3 & 4 & 0 & 2 & 1 \\
\hline \multicolumn{7}{|c|}{ Inner medulla } \\
\hline $\mathrm{MCD}$ & 3 & 4 & 4 & 1 & 1 & 1 \\
\hline VR & 3 & 4 & 4 & 2 & 4 & 4 \\
\hline $\mathrm{tLH}$ & 2 & 3 & 4 & 2 & 1 & 2 \\
\hline
\end{tabular}

Staining intensity: 0 , negative, no reactivity; 1 , trace, faint or pale brown staining with less membrane reactivity; 2 , weak, light brown staining with incomplete membrane reactivity; 3 , moderate, shaded of brown staining of intermediate darkness with usually almost complete membrane reactivity; 4 , strong, dark brown to black staining with a usually complete membrane pattern, producing a thick outline of the cell [17-19].

Aldo, aldosterone; Ep., eplerenone ( $n=6 /$ group); GL, glomerulus; PCT, proximal convoluted tubule; DCT, distal convoluted tubule; CCD, cortical collecting duct; Pcap, peritubular capillary; TALH, thick ascending limb of the loop of Henle; MCD, medullary collecting duct; VR, vasa recta; tLH, thin limb of the loop of Henle.

Eplerenone had no inhibitory effect on immunoreactivity induced by aldosterone in the VR (Fig. 3L). By contrast, the staining intensity score in the tLH was increased by pretreatment with eplerenone (Fig. 3L). The intensity score in the MCD remained at a trace level in all groups (Fig. 3K, L).

The differences in median intensity scores of cav-1 levels were significant $(P<0.05)$ by the Kruskal-Wallis test, as shown in Table 3.

\section{DISCUSSION}

The present results provide the first in vivo data simultaneously showing renal striatin and cav-1 levels and immunoreactivity 30 minutes following aldosterone administration. Aldosterone significantly enhanced the renal abundance of striatin by $50 \%$, and pretreatment with an MR antagonist, eplerenone, did not reverse that effect (Fig. 1). Previous in vitro and in vivo investigations have demonstrated that aldosterone increases striatin abundance, with a peak at 6 hours and a return to baseline after 12 hours [12]. Studies in human and mouse endothelial cells have revealed that striatin is a critical mediator of the rapid actions of aldosterone [13,22].

The precise mechanism through which aldosterone increases striatin levels has not been established. An explanation for this may be that Akt signaling induced by aldosterone enhances striatin levels. In cardiomyocytes, aldosterone provided a biphasic effect on Akt phosphorylation at either a short time (10 minutes) or a longer time (24 hours) [23]. In endothelial cells, another steroid hormone (17 $\beta$-estradiol, E2) increased striatin levels via the Akt pathway [24]. In this regard, the aldosterone-induced striatin levels in the present study may reflect the involvement of Akt signaling. In addition, E2-induced striatin expression in vascular smooth muscle cells has been shown to be related to the activation of extracellular signal-regulated kinases $1 / 2$ (ERK1/2) [25]. In an in vivo study, we previously showed that aldosterone injection increased ERK1/2 phosphorylation in rat kidney tissue at a 30-minute interval [16]. Therefore, we propose that the rapid aldosterone-induced increase in striatin levels in the rat kidney is mediated via ERK1/2 activation. In addition, a previous in vitro study in a human endothelial cell line showed that aldosterone-induced increases in striatin levels were reversed by an MR blocker, spinorolactone [12]. By con- 
Table 3. Kruskal-Wallis Analysis of Median Staining Intensity Scores of Striatin and Caveolin-1 among the Three Treatment Groups in Rat Kidney Tissue

\begin{tabular}{lllll}
\hline \multirow{2}{*}{ Areas } & \multicolumn{3}{c}{ Mean rank } & \\
\cline { 2 - 3 } & Sham & Aldo & Ep. + Aldo & \\
\hline Striatin & & & & \\
Cortex & & & & \\
GL & 3 & 12.6 & 8.4 & $0.002^{\mathrm{a}}$ \\
PCT & 4.2 & 10.6 & 9.2 & $0.029^{\mathrm{a}}$ \\
DCT & 3 & 10 & 11 & $0.005^{\mathrm{a}}$ \\
CCD & 5.8 & 12.4 & 5.8 & $0.011^{\mathrm{a}}$ \\
Pcap & 3 & 10.5 & 10.5 & $0.004^{\mathrm{a}}$ \\
Outer medulla & & & & \\
TALH & 5.9 & 5.9 & 12.2 & $0.015^{\mathrm{a}}$ \\
MCD & 5.5 & 5.5 & 13 & $0.004^{\mathrm{a}}$ \\
VR & 5 & 9.5 & 9.5 & $0.024^{\mathrm{a}}$ \\
tLH & 5 & 7.4 & 11.6 & $0.024^{\mathrm{a}}$
\end{tabular}

Inner medulla

\begin{tabular}{lcccl} 
MCD & 3.8 & 10.1 & 10.1 & $0.015^{\mathrm{a}}$ \\
VR & 5 & 9.5 & 9.5 & $0.001^{\mathrm{a}}$ \\
tLH & 3 & 9 & 12 & $0.003^{\mathrm{a}}$ \\
Cav-1 & & & & \\
Cortex & & & & \\
GL & 3 & 12.6 & 8.5 & $0.002^{\mathrm{a}}$ \\
PCT & 3 & 10.5 & 10.5 & $0.001^{\mathrm{a}}$ \\
DCT & 3 & 10.5 & 10.5 & $0.001^{\mathrm{a}}$ \\
CCD & 3 & 10.5 & 10.5 & $0.001^{\mathrm{a}}$ \\
Pcap & 3 & 10.5 & 10.5 & $0.001^{\mathrm{a}}$ \\
Outer medulla & & & & \\
TALH & 5.5 & 13 & 5.5 & $0.001^{\mathrm{a}}$ \\
MCD & 5.5 & 13 & 5.5 & $0.001^{\mathrm{a}}$ \\
VR & 8 & 8 & 8 & $1.000(\mathrm{NS})$ \\
tLH & 3 & 13 & 8 & $0.001^{\mathrm{a}}$ \\
Inner medulla & & & & \\
MCD & 8 & 8 & 8 & $1.000(\mathrm{NS})$ \\
VR & 3 & 10.5 & 10.5 & $0.001^{\mathrm{a}}$ \\
tLH & 13 & 5.5 & 5.5 & $0.001^{\mathrm{a}}$ \\
\hline
\end{tabular}

Aldo, aldosterone; Ep., eplerenone ( $n=6$ /group); GL, glomerulus; PCT, proximal convoluted tubule; DCT, distal convoluted tubule; CCD, cortical collecting duct; Pcap, peritubular capillary; TALH, thick ascending limb of the loop of Henle; MCD, medullary collecting duct; VR, vasa recta; tLH, thin limb of the loop of Henle; NS, non-significant $(n=6 /$ group).

${ }^{\mathrm{a}} P<0.05$.

trast, our present study in rat kidney tissue revealed that striatin levels remained high despite pretreatment with an MR antago- nist, eplerenone (Fig. 1). This discrepancy may be due to tissuespecific differences in responses. Further in vivo investigations are required to elucidate this issue. Thus, in the kidney, the aldosterone-induced increase in striatin levels is an MR-independent pathway.

For cav-1, the present data show that aldosterone rapidly doubled cav-1 levels in rat kidney (Fig. 1). In a previous study, aldosterone incubation induced abundant levels of cav-1 in endothelial cells [15]. In liver sinusoidal endothelial cells, aldosterone treatment increased membrane levels of cav-1 [26]. The mechanism by which aldosterone increases caveolin levels may be related to Src activation. In M-1 cell, aldosterone rapidly induced Src protein levels in a dose-dependent manner [27]. Furthermore, the abundance of Src in vascular smooth muscle cells was increased by aldosterone in a time-dependent manner (from 15 to 45 minutes) $[28,29]$. It has been noted that Src tyrosine kinases induce caveolin phosphorylation [30]. The aldosteroneinduced increase in cav-1 levels in the present study may be linked to Src activation. More in vivo studies exploring this issue are needed. In addition, incubation with PKC $\alpha$ increased cav-1 protein abundance in human lung fibroblasts [31]. Moreover, we documented that aldosterone rapidly enhanced $\mathrm{PKC} \alpha$ levels in rat kidney tissue [18]. Therefore, on the basis of the present findings, we suggest that aldosterone induces higher levels of cav-1 through PKCa activation. Of note, a previous in vitro study in vascular endothelial cells reported that aldosterone-induced changes in cav-1 levels were partially inhibited by an MR blocker, spironolactone [15]. In the present study, we used eplerenone since it has a greater selectivity for MR than spironolactone [32]. Eplerenone was also able to partially attenuate the aldosterone-induced increase in cav-1 levels in rat kidney tissue (Fig. 1). This suggests that aldosterone regulates cav1 levels, in part, through the MR pathway.

In the rapid non-genomic pathway, protein levels are actually not increased by synthesis of new molecules [9,10]. The higher levels of striatin and cav-1 in the present study may have resulted from stimulation of existing inactive proteins by various kinase enzymes, such PKC and ERK1/2. This possibility has been extensively documented in previous studies by many investigators, as well as in our experiments showing that aldosterone rapidly increased protein levels [9,10,16-19].

No previous data are available regarding the immunolocalization of striatin in rat kidney tissue. In Madin-Darby canine kidney cells, striatin was expressed in the cytosolic compartment and plasma membrane [33]. In canine cardiac myocytes, striatin was localized within the intercalated discs [34]. The present 
study is the first to document the distribution of striatin in kidney tissue. In sham rats, prominent immunoreactivity was noted in the medulla region, especially in the MCD and VR (Table 2, Fig. 2). Aldosterone rapidly increased immunostaining in most studied areas. Pretreatment with eplerenone attenuated the intensity scores in the cortex region, but immunoreactivity in the tLH and MCD was progressively enhanced. The mechanism underlying this phenomenon remains to be clarified. In the present study, cav-1 immunoreactivity showed a similar baseline distribution as in previous investigations $[35,36]$. As shown in Fig. 3, cav-1 immunostaining induced by aldosterone was obvious in the vasculature including the GL, Pcap, and VR (Table 2). The staining intensity scores in the GL, TALH, MCD, and tLH were slightly reduced by eplerenone. The distribution of striatin and cav-1 induced by the rapid action of aldosterone along nephron segments was found to show diverse patterns, implying that both proteins play significant roles in the kidney.

Of note, striatin and cav-1 are involved in modulating various cellular functions through multifunctional signals $[1,2]$. The critical role in striatin on salt-sensitive blood pressure and vascular responses has been clearly documented [3,37,38]. Unfortunately, studies of striatin related to kidney function have not been conducted. Meanwhile, cav-1 modulates health and disease through a wide range of mechanisms [39]. An in vivo study suggested that cav-1 regulates aldosterone-mediated pathways of glucose and lipid homeostasis [40]. Aldosterone-induced oxidation promoting cell defenestration takes place in cav-1-related autophagy [26]. In the kidney, cav-1 promotes renal water and salt reabsorption by modulating sodium-chloride cotransporter function and regulating vascular endothelial nitric oxide [4]. More in vivo studies are needed to elucidate the molecular mechanisms of striatin and cav-1 in modulating aldosterone pathways and, consequently, in regulating kidney function.

In conclusion, this is the first in vivo study to demonstrate that aldosterone rapidly enhances renal striatin and cav-1 levels. Aldosterone increases striatin levels by an MR-independent pathway, whereas cav-1 is partially regulated through MR.

\section{CONFLICTS OF INTEREST}

No potential conflict of interest relevant to this article was reported.

\section{ACKNOWLEDGMENTS}

This research was supported by Grant no. RA61/055 from the
Ratchadapiseksompoth Research Fund, Faculty of Medicine, and the 90th Anniversary of Chulalongkorn University Fund (2/2562, Ratchadapiseksompoth Research Fund), Chulalongkorn University.

\section{AUTHOR CONTRIBUTIONS}

Conception or design: S.E.O. Acquisition, analysis, or interpretation of data: K.I., S.E.O. Drafting the work or revising: K.I., S. E.O. Final approval of the manuscript: K.I., K.M., S.E.O., S.E.O.

\section{ORCID}

Kevalin Inthachart https://orcid.org/0000-0001-5668-9300

Somchit Eiam-Ong https://orcid.org/0000-0001-8646-4348

\section{REFERENCES}

1. Hwang J, Pallas DC. STRIPAK complexes: structure, biological function, and involvement in human diseases. Int $\mathrm{J}$ Biochem Cell Biol 2014;47:118-48.

2. Baudrand R, Pojoga LH, Romero JR, Williams GH. Aldosterone's mechanism of action: roles of lysine-specific demethylase 1, caveolin and striatin. Curr Opin Nephrol Hypertens 2014;23:32-7.

3. Garza AE, Rariy CM, Sun B, Williams J, Lasky-Su J, Baudrand R, et al. Variants in striatin gene are associated with salt-sensitive blood pressure in mice and humans. Hypertension 2015;65:211-7.

4. Williere Y, Borschewski A, Patzak A, Nikitina T, Dittmayer $\mathrm{C}$, Daigeler AL, et al. Caveolin 1 promotes renal water and salt reabsorption. Sci Rep 2018;8:545.

5. Bollag WB. Regulation of aldosterone synthesis and secretion. Compr Physiol 2014;4:1017-55.

6. Connell JM, Davies E. The new biology of aldosterone. J Endocrinol 2005;186:1-20.

7. Grossmann C, Gekle M. New aspects of rapid aldosterone signaling. Mol Cell Endocrinol 2009;308:53-62.

8. Falkenstein E, Wehling M. Nongenomically initiated steroid actions. Eur J Clin Invest 2000;30:51-4

9. Thomas W, Harvey BJ. Mechanisms underlying rapid aldosterone effects in the kidney. Annu Rev Physiol 2011;73:33557.

10. Dooley R, Harvey BJ, Thomas W. Non-genomic actions of aldosterone: from receptors and signals to membrane targets. Mol Cell Endocrinol 2012;350:223-34.

www.e-enm.org 
11. Tsugita M, Iwasaki Y, Nishiyama M, Taguchi T, Shinahara M, Taniguchi Y, et al. Glucocorticoid receptor plays an indispensable role in mineralocorticoid receptor-dependent transcription in GR-deficient BE(2)C and T84 cells in vitro. Mol Cell Endocrinol 2009;302:18-25.

12. Pojoga LH, Coutinho P, Rivera A, Yao TM, Maldonado ER, Youte R, et al. Activation of the mineralocorticoid receptor increases striatin levels. Am J Hypertens 2012;25:243-9.

13. Coutinho P, Vega C, Pojoga LH, Rivera A, Prado GN, Yao TM, et al. Aldosterone's rapid, nongenomic effects are mediated by striatin: a modulator of aldosterone's effect on estrogen action. Endocrinology 2014;155:2233-43.

14. Pojoga LH, Romero JR, Yao TM, Loutraris P, Ricchiuti V, Coutinho P, et al. Caveolin-1 ablation reduces the adverse cardiovascular effects of N-omega-nitro-L-arginine methyl ester and angiotensin II. Endocrinology 2010;151:1236-46.

15. Igarashi J, Hashimoto T, Shoji K, Yoneda K, Tsukamoto I, Moriue T, et al. Dexamethasone induces caveolin-1 in vascular endothelial cells: implications for attenuated responses to VEGF. Am J Physiol Cell Physiol 2013;304:C790-800.

16. Sinphitukkul K, Eiam-Ong S, Manotham K, Eiam-Ong S. Nongenomic effects of aldosterone on renal protein expressions of pEGFR and pERK1/2 in rat kidney. Am J Nephrol 2011;33:111-20.

17. Eiam-Ong S, Sinphitukkul K, Manotham K, Eiam-Ong S. Rapid nongenomic action of aldosterone on protein expressions of Hsp90 ( $\alpha$ and $\beta$ ) and pc-Src in rat kidney. Biomed Res Int 2013;2013:346480.

18. Eiam-Ong S, Sinphitukkul K, Manotham K, Eiam-Ong S. Rapid action of aldosterone on protein expressions of protein kinase $\mathrm{C}$ alpha and alphal sodium potassium adenosine triphosphatase in rat kidney. J Steroids Horm Sci 2014;5:125.

19. Eiam-Ong S, Chaipipat M, Manotham K, Eiam-Ong S. Rapid action of aldosterone on protein levels of sodium-hydrogen exchangers and protein kinase $\mathrm{C}$ beta isoforms in rat kidney. Int J Endocrinol 2017;2017:2975853.

20. Chen YK, Chen CY, Hu HT, Hsueh YP. CTTNBP2, but not CTTNBP2NL, regulates dendritic spinogenesis and synaptic distribution of the striatin-PP2A complex. Mol Biol Cell 2012;23:4383-92.

21. Zhang J, Jiang Z, Bao C, Mei J, Zhu J. Cardiopulmonary bypass increases pulmonary microvascular permeability through the Src kinase pathway: involvement of caveolin-1 and vascular endothelial cadherin. Mol Med Rep 2016;13:2918-24.

22. Machado-Fiallo ED, Vega C, Ramos-Rivera A, Benabe-Carlo JA, Prado GN, Pojoga LH, et al. Striatin is a novel regula- tor of aldosterone-mediated endothelial cell activation. Blood 2013;122:3523.

23. Nagoshi T, Date T, Fujisaki M, Yoshino T, Sekiyama H, Ogawa K, et al. Biphasic action of aldosterone on Akt signaling in cardiomyocytes. Horm Metab Res 2012;44:931-7.

24. Zheng S, Sun P, Liu H, Li R, Long L, Xu Y, et al. 17 $\beta$-Estradiol upregulates striatin protein levels via Akt pathway in human umbilical vein endothelial cells. PLoS One 2018;13:e0202500.

25. Zheng S, Chen X, Hong S, Long L, Xu Y, Simoncini T, et al. $17 \beta$-Estradiol inhibits vascular smooth muscle cell migration via up-regulation of striatin protein. Gynecol Endocrinol 2015;31:618-24.

26. Luo X, Dan Wang, Luo X, Zhu X, Wang G, Ning Z, et al. Caveolin 1-related autophagy initiated by aldosterone-induced oxidation promotes liver sinusoidal endothelial cells defenestration. Redox Biol 2017;13:508-21.

27. Braun S, Losel R, Wehling M, Boldyreff B. Aldosterone rapidly activates Src kinase in M-1 cells involving the mineralocorticoid receptor and HSP84. FEBS Lett 2004;570:69-72.

28. Callera GE, Montezano AC, Yogi A, Tostes RC, He Y, Schiffrin EL, et al. c-Src-dependent nongenomic signaling responses to aldosterone are increased in vascular myocytes from spontaneously hypertensive rats. Hypertension 2005;46:10328.

29. Callera GE, Touyz RM, Tostes RC, Yogi A, He Y, Malkinson $\mathrm{S}$, et al. Aldosterone activates vascular p38MAP kinase and NADPH oxidase via c-Src. Hypertension 2005;45:773-9.

30. Li S, Seitz R, Lisanti MP. Phosphorylation of caveolin by src tyrosine kinases. The alpha-isoform of caveolin is selectively phosphorylated by v-Src in vivo. J Biol Chem 1996;271:38638.

31. Tourkina E, Gooz P, Pannu J, Bonner M, Scholz D, Hacker S, et al. Opposing effects of protein kinase Calpha and protein kinase Cepsilon on collagen expression by human lung fibroblasts are mediated via MEK/ERK and caveolin-1 signaling. J Biol Chem 2005;280:13879-87.

32. Jaisser F, Farman N. Emerging roles of the mineralocorticoid receptor in pathology: toward new paradigms in clinical pharmacology. Pharmacol Rev 2016;68:49-75.

33. Breitman M, Zilberberg A, Caspi M, Rosin-Arbesfeld R. The armadillo repeat domain of the APC tumor suppressor protein interacts with Striatin family members. Biochim Biophys Acta 2008;1783:1792-802.

34. Meurs KM, Mauceli E, Lahmers S, Acland GM, White SN, Lindblad-Toh K. Genome-wide association identifies a deletion in the 3 ' untranslated region of striatin in a canine model 
of arrhythmogenic right ventricular cardiomyopathy. Hum Genet 2010;128:315-24.

35. Fujigaki Y, Sakakima M, Sun Y, Goto T, Ohashi N, Fukasawa $\mathrm{H}$, et al. Immunohistochemical study on caveolin-1alpha in regenerating process of tubular cells in gentamicin-induced acute tubular injury in rats. Virchows Arch 2007;450:671-81.

36. Breton S, Lisanti MP, Tyszkowski R, McLaughlin M, Brown D. Basolateral distribution of caveolin-1 in the kidney. Absence from $\mathrm{H}+$-atpase-coated endocytic vesicles in intercalated cells. J Histochem Cytochem 1998;46:205-14.

37. Garza AE, Pojoga LH, Moize B, Hafiz WM, Opsasnick LA, Siddiqui WT, et al. Critical role of striatin in blood pressure and vascular responses to dietary sodium intake. Hyperten- sion 2015;66:674-80.

38. Gupta T, Connors M, Tan JW, Manosroi W, Ahmed N, Ting PY, et al. Striatin gene polymorphic variants are associated with salt sensitive blood pressure in normotensives and hypertensives. Am J Hypertens 2017;31:124-31.

39. Cohen AW, Hnasko R, Schubert W, Lisanti MP. Role of caveolae and caveolins in health and disease. Physiol Rev 2004; 84:1341-79.

40. Baudrand R, Gupta N, Garza AE, Vaidya A, Leopold JA, Hopkins PN, et al. Caveolin 1 modulates aldosterone-mediated pathways of glucose and lipid homeostasis. J Am Heart Assoc 2016;5:e003845. 